\title{
Facile Synthesis of High Lateral Graphene Oxide Sheets for Visible Light-driven Photocatalytic Degradation of Industrial Dyes towards Water Treatment Applications
}

Jagpreet Singh

Sri Guru Granth Sahib World University

Harpreet Kaur

Sri Guru Granth Sahib World University

Khalid Mujasam Batoo ( $\sim$ kbatoo@ksu.edu.sa )

King Saud University

Sanjeev Kumar

Sri Guru Granth Sahib World University

Mohit Rawat

Sri Guru Granth Sahib World University

Syed Adil Farooq

King Saud University

Emad H. Raslan

King Saud University

Saravanan Pandiarajan

King Saud University

Nirmala Grace Andrews

Vellore Institute of Technology University

Muthumareeswaran MR

King Saud University

\section{Research Article}

Keywords: Graphene oxide, Dye degradation, Photocatalysis, environmental remediation, wastewater treatment

Posted Date: December 21st, 2020

DOI: https://doi.org/10.21203/rs.3.rs-127571/v1 
License: (c) (i) This work is licensed under a Creative Commons Attribution 4.0 International License. Read Full License 


\section{Facile Synthesis of High Lateral Graphene Oxide Sheets for Visible}

Light-driven Photocatalytic Degradation of Industrial Dyes towards water treatment applications

Jagpreet Singh ${ }^{1}$, Harpreet Kaur $^{2}$, Khalid Mujasam Batoo ${ }^{3, *}$, Sanjeev Kumar, ${ }^{2, * *}$, Mohit Rawat $^{1, * *}$, Syed Adil Farooq ${ }^{4}$, Emad H. Raslan ${ }^{5}$, Saravanan Pandiarajan ${ }^{6}$, Andrews Nirmala Grace ${ }^{7}$, Muthumareeswaran $\mathrm{MR}^{3}$

${ }^{1}$ Department of Nanotechnology, Sri Guru Granth Sahib World University, Fatehgarh Sahib - 140406, Punjab, India

${ }^{2}$ Department of Physics, Sri Guru Granth Sahib World University, Fatehgarh Sahib 140406, Punjab, India

${ }^{3}$ King Abdullah Institute for Nanotechnology, King Saud University, P.O. Box 2455, Riyadh-11451, Saudi Arabia

${ }^{4}$ Department of Chemistry, College of Science, King Saud University, PO Box 2455, Riyadh-11451, Saudi Arabia

${ }^{5}$ Department of Physics, College of Science, King Saud University, PO Box 2455, Riyadh-11451, Saudi Arabia

${ }^{6}$ Department of Self Development Skills, CFY Deanship, King Saud University, Riyadh, Saudi Arabia

${ }^{7}$ Centre for Nanotechnology Research (CNR), Vellore Institute of Technology, Vellore - 632014, Tamil Nadu, India

Corresponding author: kbatoo@ksu.edu.sa

Co-corresponding authors**: mohitnano.nit@gmail.com, kumarsanju25@gmail.com 


\begin{abstract}
In this work, a facile synthesis of multilayer graphene oxide (GO) sheets having a twodimensional structure has been realized using the modified Hummers and Offeman method. The as-synthesized GO was analyzed by UV-visible, FTIR, Powder X-ray Diffraction (PXRD), HRTEM, FESEM, and EDX for optical, chemical, structural, morphological, topographical, and elemental analysis respectively. The results reveal that GO shows an absorption band at $232 \mathrm{~nm}$. The FTIR spectrum shows the oxygenrich groups in GO, and PXRD confirms the major GO peak at $10.35^{\circ}$ along with few minor peaks. HRTEM and FESEM confirm the two-dimensional GO sheets along with high lateral dimensions. The as-synthesized GO with a number of available functional groups and high lateral dimension was efficiently used for the photocatalytic degradation of Coralline Red BS (CR BS) and Reactive Blue 81 (RB81) dyes. This study reveals that, compared to graphene, pristine GO sheets significantly influence the degradation of CR BS and RB81 dyes. This work significantly contributes to the use of pristine GO for the removal of toxic dyes from wastewater. The evaluation of the dye degradation rate and GO reusability along with the kinetic studies is explained in detail.
\end{abstract}

Keywords: Graphene oxide; Dye degradation; Photocatalysis, environmental remediation, wastewater treatment 


\section{Introduction}

Nanomaterials offer unique physical properties and have attracted tremendous attention from researchers and scientists. The facile synthesis of nanoparticles and system production increased the understanding of nanoscience with an interface from various research fields including physics, chemistry, and material sciences ${ }^{1}$. The advancement in nanotechnology has led to the synthesis of novel nanomaterials that find effective use in advanced applications, such as nanoelectronics, nanomedicine, and customer products $^{2}$. Carbon, a first-row element on the periodic table is widely available in three forms, namely diamond, graphite, and fullerenes. Graphene, an allotrope of carbon is the thinnest, strongest, and stiffest compound and has unique properties, such as being an excellent conductor of heat and electricity. Single-layer graphene that is only oneatom-thick has received a huge attracting attention because of its potential to provide extraordinary thermal, electrical, and optical properties in material science, biochemistry, and medicine ${ }^{3}$. Currently, the major research topics in materials sciences are the development of other allotropes of graphene ${ }^{4}$ because of its unique properties allowing its vast application in various fields ${ }^{5}$ like (i) in graphene, its electron transportation is labeled by Dirac's equation, allowing access to quantumelectrodynamics as part of solid-state physics. (ii) graphene-based systems are stable because of the high transport of electrons through the surface at ambient-temperature embedded with mechanical stability. (iii) Various aspects of graphite and nanotubes can be regarded as an interface of graphene.

The great interest in graphene oxide (GO) might be because of its inexpensive manufacturing rate and extensive capability to obtain from graphene ${ }^{6}$, and its current scalability is a desired feature. After oxidation, graphite's carbon layers are filled with 
oxygen molecules and converted to GO. GO is a side product during oxidation of graphite and the obtained GO has high interplanar spacing between the layers as compared to graphite. After oxidation, it spreads in a base solution such as water ${ }^{7}$ and finally the carbon layers are reduced and separated into specific layers. During these processes, massive quantities of functional groups (oxygen-containing) have been introduced onto the individual sides of the graphite sheet. The implantation of the functional groups overpowers the van-der Waals force between the sheets, increasing the interlayer spacing. The sonication easily pulls the structure and expands it further to its maximum, exfoliating the graphite into multilayer or single-layer sheets separation. The obtained multilayer GO sheets find potential applications in elastic optoelectronics, such as organic light-emitting diodes and solar cells ${ }^{8,9}$. The basic requirement for these applications besides its high conductive and transparent nature, is that the electrode materials must be lightweight, flexible, low-cost, and compatible with large-scale manufacturing.

In this study, the authors described the facile synthesis of GO sheets using Hummer's method and characterized it for its structural, optical, and photochemical properties ${ }^{10}$. Additionally, the photocatalytic activity of the as-synthesized GO for the degradation of Reactive blue 81 (RB81) and Coralline red (CR BS) dyes under direct sunlight is investigated in detail. The process of photocatalysis is achieved by photons with threshold energy greater than or equal to the bandgap energy semiconductor. An incident photon excites an electron from the valence band (VB) to the conduction band (CB), creating a positive hole in the VB, probably leading to the formation of hydroxyl radicals when reacting with hydroxyl-ion in water, which is finally available for oxidation. Simultaneously in the $\mathrm{CB}$, an excited electron reduces the oxygen and acts as 
an oxidizing agent. One of the main drawbacks observed is the recombination of the photo-generated electron with their hole ${ }^{11-13}$. This phenomenon dissipates almost all the photon energy resulting in the low-efficiency of photocatalysis. Therefore, the development of a reliable and efficient photocatalyst is always desired, and critical in this study.

\section{Materials and Methods}

All the chemical-compounds and reagents used were of analytical-reagent grade and were practiced as procured without any additional refinement. Graphite powder $(\geq 99 \%)$, Sodium nitrate $\left(\mathrm{NaNO}_{3} ; \geq 98 \%\right)$, Potassium permanganate $\left(\mathrm{KMnO}_{4} ; \geq 99 \%\right)$, Hydrogen peroxide $\left(\mathrm{H}_{2} \mathrm{O}_{2} ; \geq 40 \%\right)$, Sulfuric acid $\left(\mathrm{H}_{2} \mathrm{SO}_{4} ; \geq 98 \%\right)$, and Hydrochloric acid ( $\mathrm{HCl}$; $\geq 35 \%$ ) were purchased respectively form Asbury Graphite Mill Inc. US, Nice Chemicals Pvt. Ltd. India, Ramagundam Fertilizers and Chemicals Ltd. India, Merck India, ACS Chemicals India, and RANKEM Chemicals India.

The most promising and widely used technique for the preparation of GO is Hummers and Offeman's $(1958)^{14,15}$. In this designed synthesis strategy, the authors can easily control the structural, morphological and optical properties by changing the oxidizing agents used to exfoliate graphite powder. For a typical experiment process, the graphite flakes and $\mathrm{NaNO}_{3}$ in 1:1 ratio were taken in $45 \mathrm{ml}$ of $\mathrm{H}_{2} \mathrm{SO}_{4}$. The mixture-solution was reserved underneath ice immersion $\left(0-5^{\circ} \mathrm{C}\right)$ with constant stirring for five hours. After that, a definite amount of oxidizing agent $\left(\mathrm{KMnO}_{4}\right)$ was gently added to this suspension to generate the graphite solution. The addition of $\mathrm{KMnO}_{4}$ was sensibly supervised to keep the reaction-temperature below $15^{\circ} \mathrm{C}$. The mixed chemical solution was stirred for another two hours and diluted by gently adding $92 \mathrm{ml}$ of water. After removal from the ice-bath, the solution was stirred at $40^{\circ} \mathrm{C}$ until it turned deep red-brown ${ }^{7}$. The solution 
was held in a reflux system at $98^{\circ} \mathrm{C}$ for $10-15$ minutes. After this, the solution was mixed with $10 \mathrm{ml} \mathrm{H}_{2} \mathrm{O}_{2}$ to stop any further reaction. A huge amount of bubbles was released and the shade of the solution was transformed into bright yellow. The colored suspension was filtered and washed for purification with $10 \%$ of $\mathrm{HCl}$ and repeatedly with deionized water. Finally, the product was filtered and dried in a vacuum oven at $50^{\circ} \mathrm{C}$ and flakes of $\mathrm{GO}$ were obtained in the powder form.

RB81 and CR BS dyes procured from Parshwanath Dye Stuff Industries, Ahmedabad, India, were cast-off as a standard pollutant to evaluate the photocatalytic action of the as-synthesized GO sample. Sodium hydroxide $(\mathrm{NaOH})$ and $\mathrm{HCl}$, obtained from HiMedia Laboratory Private Limited, Mumbai, India, were used to adjust the $\mathrm{pH}$ of the solutions for the photocatalytic test. The peak intensity at the highest absorbance wavelength (581 nm and $415 \mathrm{~nm}$ ) was monitored during the photocatalytic experiments to evaluate the degradation of $\mathrm{RB} 81$ and $\mathrm{CR}$ BS dyes respectively in the unexposed/exposed GO samples. For a standard photocatalytic test, a $200 \mathrm{~mL}$ solution was prepared by dispersing $1 \mathrm{mML}^{-1}$ of dye (RB81 or CR BS) in de-ionized water and $0.4 \mathrm{gL}^{-1}$ of $\mathrm{GO}$ was added under magnetic-stirring and ultra-sonication. After that, the suspension $\mathrm{pH}$ was set between 4 to 11 and stirred for 30 hours in the dark to establish an adsorption/desorption equilibrium. The solution was then exposed to sunlight for 90 minutes under stirring.

During this process, in a systematic interval of 15 minutes, a sample of $5 \mathrm{~mL}$ from the aqueous suspension solution was taken, centrifuged (10000 rpm), and the supernatant (dye solution) was examined with a UV-vis spectrophotometer. The photocatalytic activity of GO was calculated using the following equation:

$$
\text { Percentage degradation of dye }=\left(\frac{C_{0}-C_{t}}{C_{t}}\right) \times 100
$$


where $C_{0}$ is the starting dye concentration, and $C_{\mathrm{t}}$ the concentration of dye after exposure to direct sunlight for time $t(0,15,30,45,60,75$, and 90 minutes).

The phase purity and the structure identification of the synthesized GO powder was determined from PXRD patterns, performed using the PAN analytical X-ray diffractometer. HF-3300 and Transmission Electron Microscope (TEM) powered by Hitachi's recorded provided the sample morphology. The UV-visible absorbance spectrum of the sample was done using Shimadzu UV 2600 spectrophotometer. The IR spectrum of the GO sample was analyzed and evaluated using the Fourier Transformed Infrared (FTIR) spectrometer powered by Bruker Alpha. The elemental configuration and chemical purity were investigated using an energy beam of $20 \mathrm{keV}$ using Oxford instruments EDX.

\section{Results and discussions}

\subsection{UV-Visible absorption analysis}

The UV-vis spectrum of standard GO displays a quantitative feature that could be used as an identification tool to confirm the synthesis of GO visually. Figure 1 depicts the UV-vis optical spectrum of the as-synthesized GO. The spectrum shows that GO exhibit a high absorption at a wavelength of $232 \mathrm{~nm}$ and a shoulder peak at $300 \mathrm{~nm}$. The low and high wavelength peaks are instigated from the $\pi-\pi^{*}$ transition of aromatic $\mathrm{C}-\mathrm{C}$ bonds and the $n-\pi *$ transition of the $\mathrm{C}=\mathrm{O}$ bonds, respectively ${ }^{16}$. These findings show that carbonyl groups are present on GO surface. Furthermore, the spectrum shows that GO absorb in the visible region and possesses a high absorption in the UV-vis range. This could result in a good photo response of the as-synthesized GO sample in the visible range, leading to a wide possibility for different applications. 


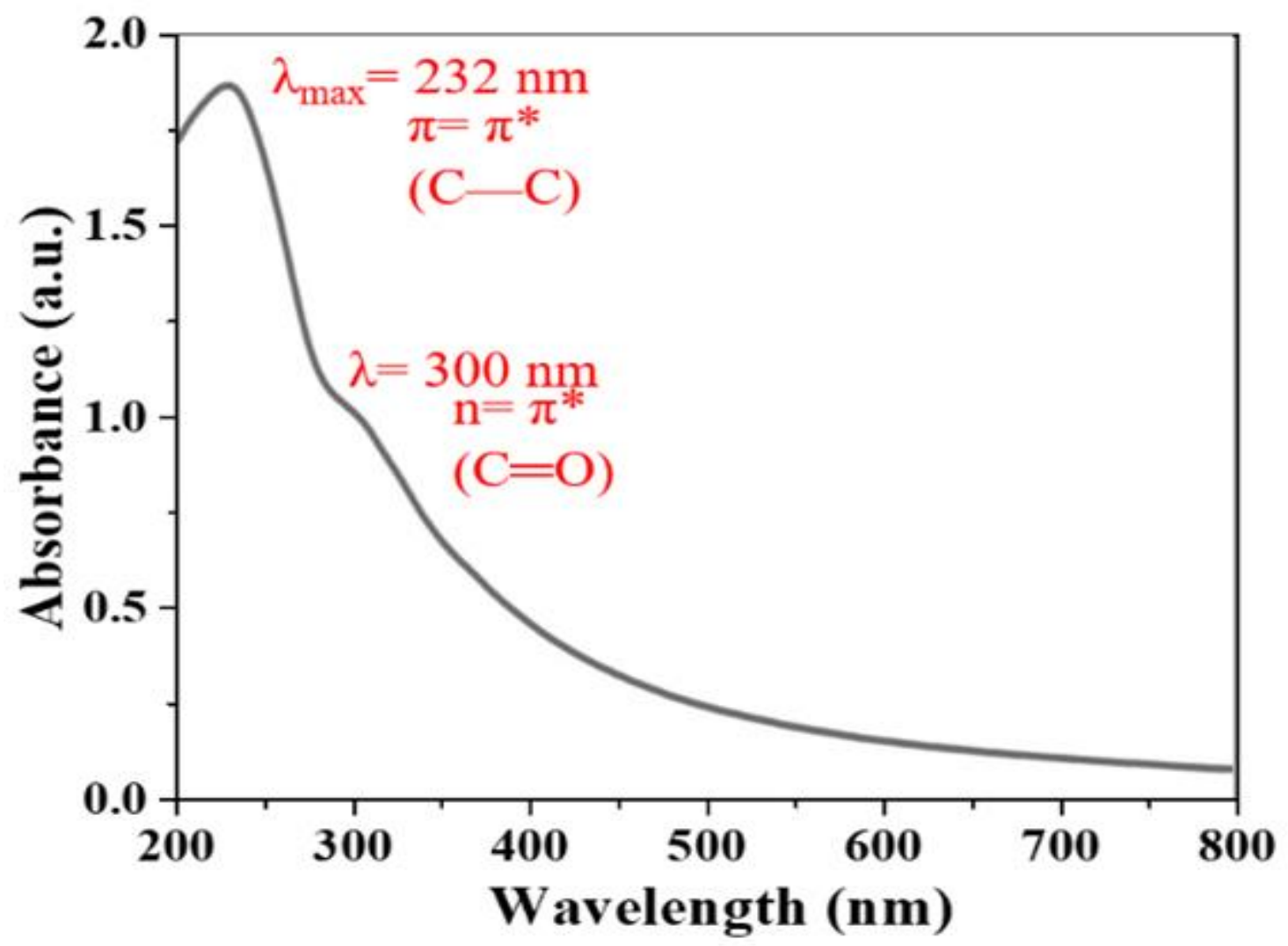

Figure 1. UV-Vis absorption spectrum of GO.

\subsection{FTIR analysis}

FTIR measurement was conducted to examine the bonding-interactions in the synthesized GO. Figure 2 shows a typical FTIR spectrum of GO and result justifies the effective oxidation of graphite. The major absorption band at $669 \mathrm{~cm}^{-1}$ belongs to the $\mathrm{C}$ O-C of epoxy groups. Another band at $1104 \mathrm{~cm}^{-1}$ is corresponding to the stretching of the $\mathrm{C}-\mathrm{O}$ bond, indicating the presence of oxide functional groups after an oxidation course. The peaks at $1443 \mathrm{~cm}^{-1}$ and $1594 \mathrm{~cm}^{-1}$ are due to the bending vibration of the O-H groups and the stretching/bending motions of (skeletal vibrations) unoxidized graphite atoms, respectively ${ }^{17,18}$. The strong $\mathrm{C}=\mathrm{O}$ peak at $1740 \mathrm{~cm}^{-1}$ attributes to the stretching-vibrations of carboxylic and carbonyl groups. Two absorption bands at 2360 $\mathrm{cm}^{-1}$ and $2858 \mathrm{~cm}^{-1}$ appeared because of the stretching of the $\mathrm{C}-\mathrm{H}$ bond and show the 
successful splicing of the $\mathrm{O}_{2}$-based functional groups on the graphite sheet's surface and edges. The region from $2928 \mathrm{~cm}^{-1}$ to $3772 \mathrm{~cm}^{-1}$ showing a broad peak supports the stretching of the $\mathrm{O}-\mathrm{H}$ bond of water molecules ${ }^{19}$.

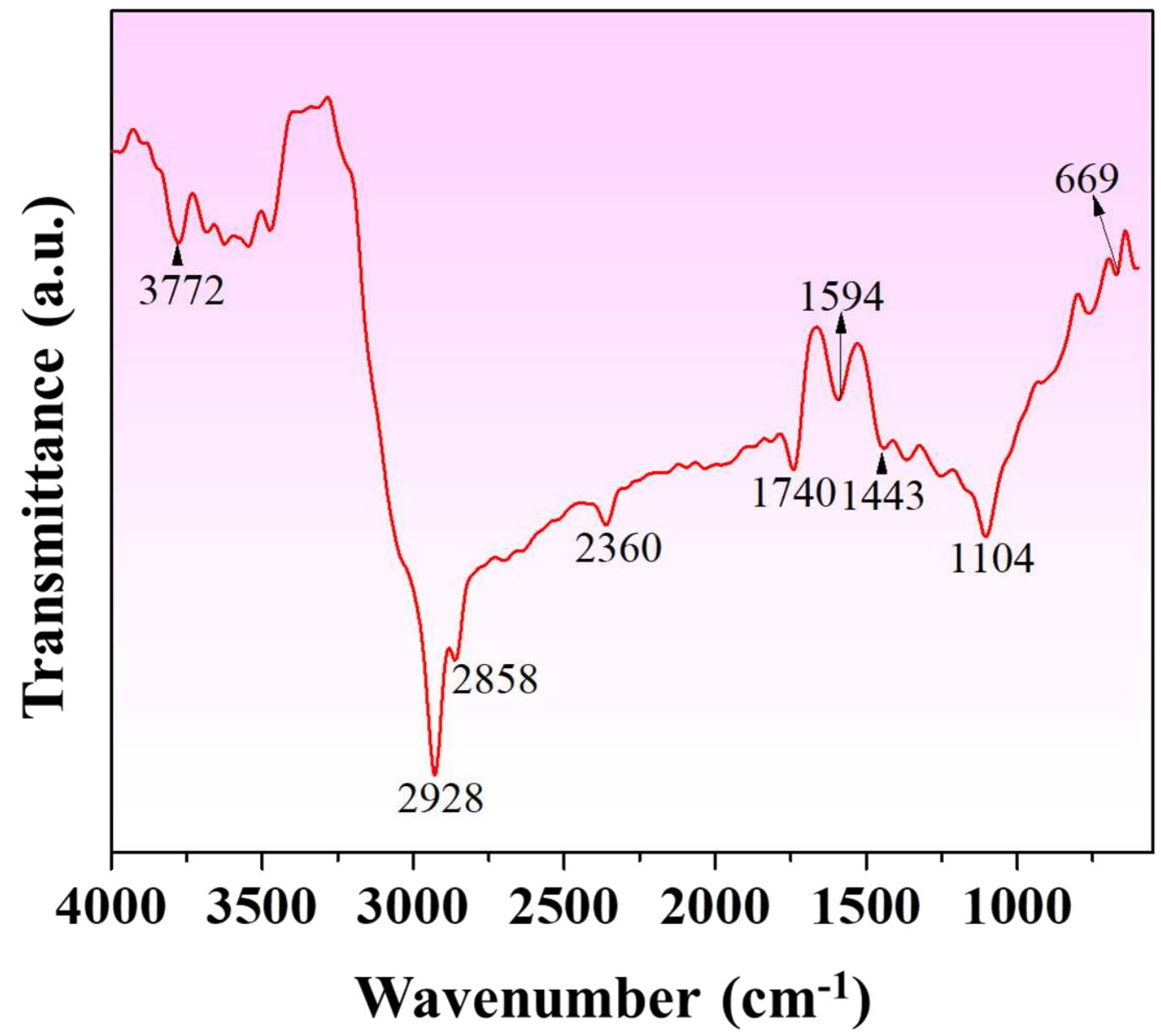

Figure 2. FT-IR spectrum of graphene oxide.

The most notable vibrations possessing the broad and intense peaks of $\mathrm{O}-\mathrm{H}$ groups centered at $3400 \mathrm{~cm}^{-1}$ are related to dissimilar $\mathrm{O}_{2}$ functionalities on GO. The FTIR findings suggest the existence of various types of $\mathrm{O}_{2}$ functionalities on GO.

\subsection{Structural and Morphological analysis}

Figure 3 shows the PXRD pattern of the GO sample recorded in the $2 \theta$ long-range of 5$60^{\circ}$ along with standard graphite diffraction peaks (inset Fig. 3, JCPDS 460870). The 
as-synthesized GO displays a high-intensity reflection peak at $2 \theta, 10.35^{\circ}$ matching the (001) plane, and low-intensity reflection peak at $2 \theta, 42.41^{\circ}$ matching the (100) plane of GO. Along with the GO diffraction peaks, the traces of resources of graphite flakes used to synthesize GO are observed at $26.52^{\circ}$ (marked as asterisk*), corresponding to the (002) plane of graphite (Inset Fig. 3, JCPDS 460870).

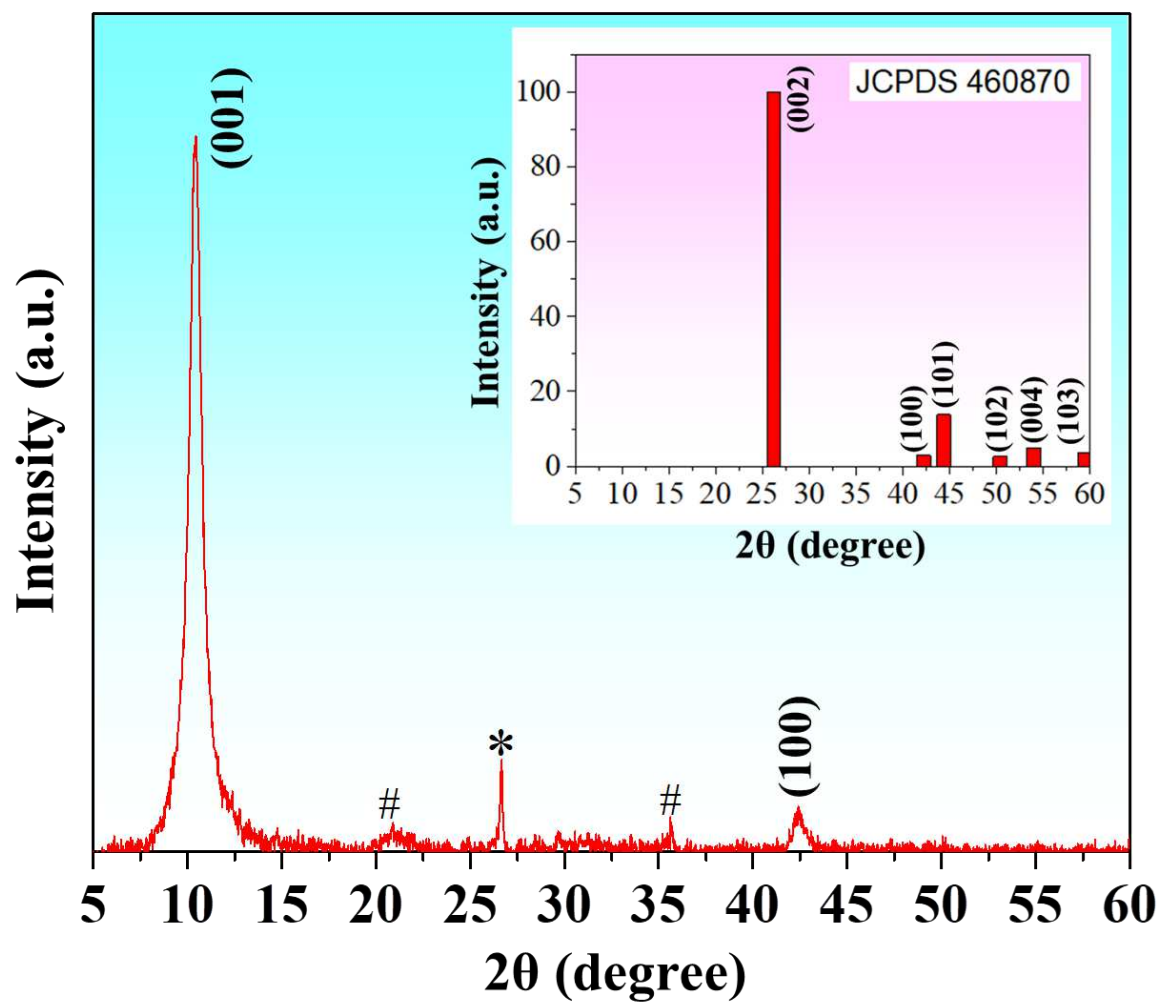

Figure 3. Powder X-Ray diffraction spectrum of the as synthesized GO.

The appearance of this peak proposes that the GO is not oxidized completely. One could deduce that with oxidation, the graphite flake's (002) plane is shifted from the higher scattering angle $\left(2 \theta=26.52^{\circ}\right)$ to the lower angle $\left(2 \theta=10.35^{\circ}\right)$. Two trivially small peaks are detected at $2 \theta 21.32^{\circ}$ and $35.69^{\circ}$ (marked as hashtag \#) in the PXRD pattern, showing that carbon is not fully interconnected with the oxygen atoms ${ }^{20}$.

Separation in-between sets of parallel planes (interplanar spacing) are calculated from the Bragg's diffraction equation as follows: 


$$
2 d \operatorname{Sin} \theta=n \chi
$$

where $d$ is the separation in-between parallel planes, $\theta$ is Bragg's angle, $n$ is the order of reflection, and $\chi$ is the X-ray wavelength. Using this, $d$ is calculated as $8.51 \AA$ for GO, which is quite large compared to pristine graphite interplanar spacing (3.69 ̊). This could be because of the presence of oxygen-containing functional groups in-between graphite layers happened during the oxidation process and injected water molecules ${ }^{21}$. Based on the XRD analysis, GO is the primary material obtained in the synthesis compared to virgin graphite. Therefore, GO could be used in those applications that requires large surface-to-volume ratio or sensitive electronic properties.

Figure 4 shows the TEM micrographs of the as-synthesized GO and the micrographs show sheet-like morphology with different transparencies of the investigated area.

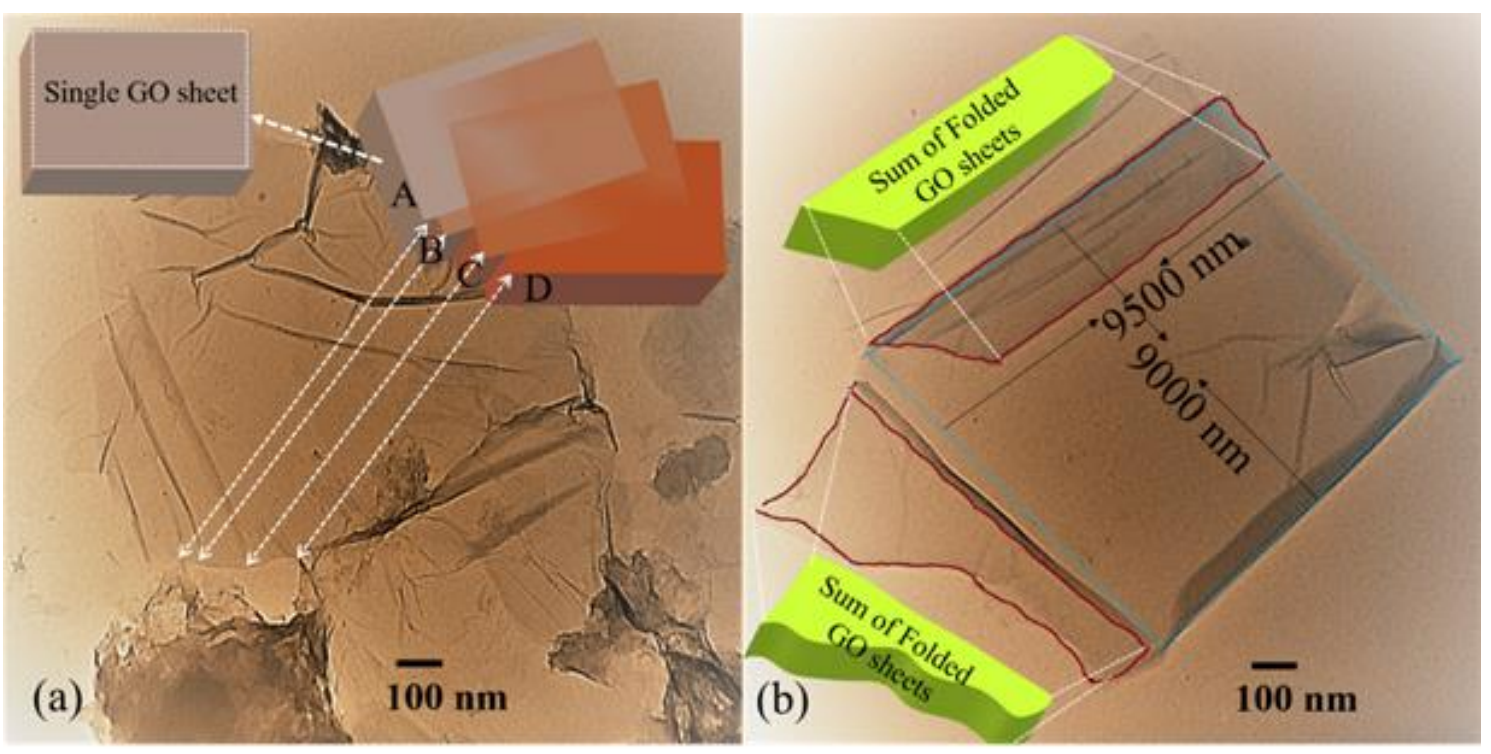

Figure 4. TEM images of graphene oxide.

Images expose the existence of the number of overlapped sheets with sideway sizes ranging from several $\mathrm{nm}$ to several $\mu \mathrm{m}$, confirming the complete exfoliation of the GO down to individual GO sheets. 
Figure 4(a) also shows that the sheets are plane, light, translucent, and stacked together. The animated part shows a single GO sheet for better depiction along with the sheets that are stacked together. The shadowy zones designate the dense stacking in the structure of GO and confirm the existence of graphene layers containing some oxygenrich functional groups ${ }^{22}$. The designated bright areas confirm that the thinner films comprises of few GO because of the exfoliation of graphene. Figure 4(b) displays the well-oriented but stacked pieces with sizes of their lateral dimensions. The crinkles between the sheets and the twists at the boundaries are observed, which belong to a large number of defects and functional groups that appeared during oxidation ${ }^{23}$. The schematics of the crinkle edges are also highlighted.

In another observation, such shady areas belong to the large thickness of the sheets formed because of stacking as compared to single-layer pristine graphene. In general, this confirms the existence of oxygen groups embedded in the adjacent graphene sheet, which could lead to atomic-scale roughness arising from structural imperfections $\left(\mathrm{sp}^{3}\right.$ hybridization) produced on the atomically even graphene sheet ${ }^{24}$. The characteristics of graphene are associated with individual sheets; however, notably, a few stacked GO layers had a higher adsorption capacity compared to today's nanomaterials or pristine GO sheets in the degradation and removal of certain heavy and toxic metal ions from aqueous dispersion ${ }^{22-24}$. Moreover, oxygen groups on top of the surfaces of GO are critical in metal-ion sorption. In this work, FESEM and EDX were used to explore the topography of the GO sample and elemental composition information. 


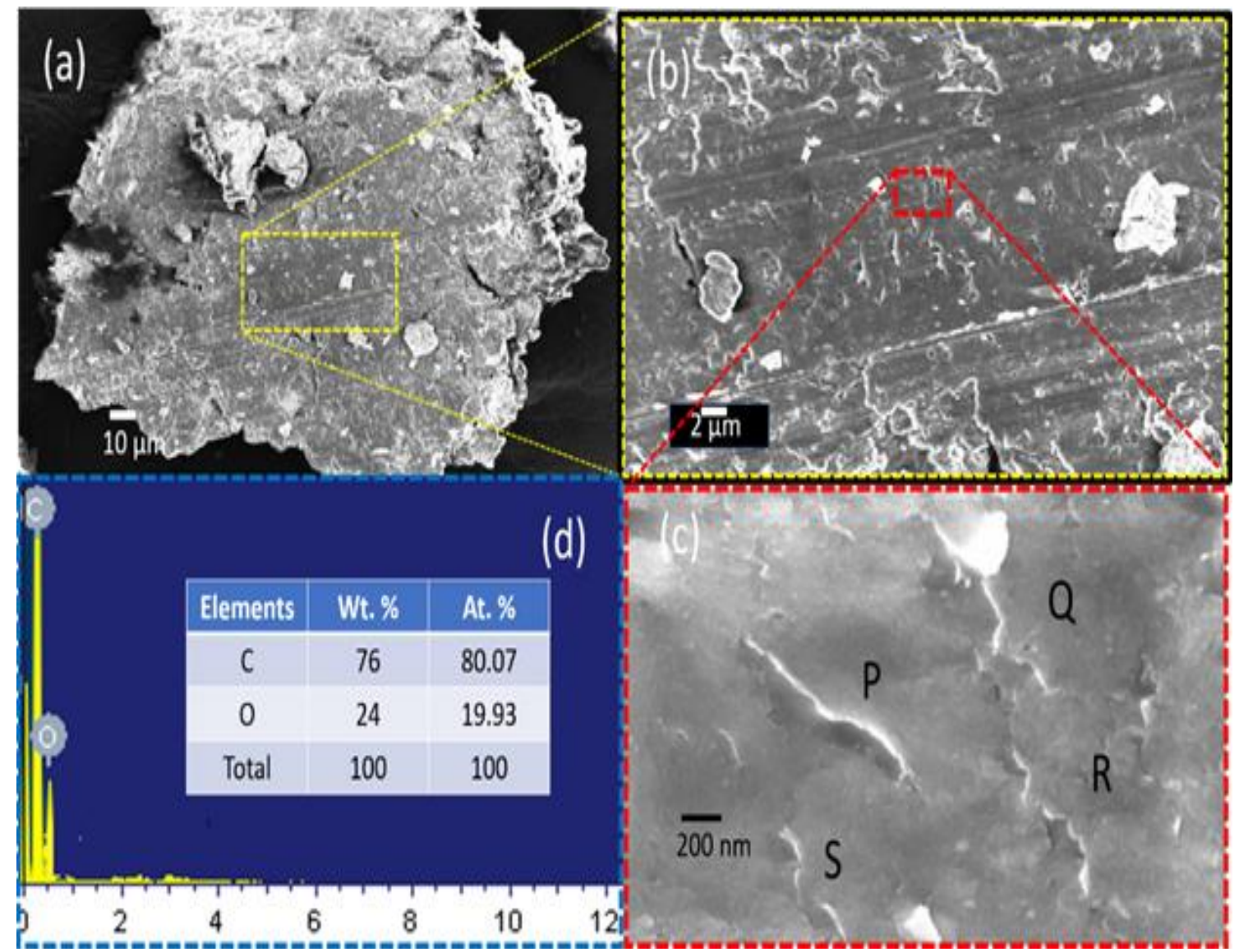

Figure 5. FESEM of Graphene Oxide by Hummers method and EDX pattern.

Figure 5(a) shows the FESEM micrographs of the as-synthesized GO sample at a scale of $10 \mu \mathrm{m}$. The highlighted cross-section (yellow doted) has been magnified, showing some grains, boundaries and aggregates of GO (Figure. 5(b)). Further magnification ( $\mu \mathrm{m}$ to $\mathrm{nm}$ scale) was done (red dotted cross-section) to closely examine the grains (Figure. 5(c)), which shows well-defined, randomly oriented, and crumpled twodimensional GO sheets. The sheets have discontinuous ends and lateral dimensions to a few $\mu \mathrm{m}$. Regions $\mathrm{P}, \mathrm{Q}, \mathrm{R}$, and $\mathrm{S}$ show the four individual overlapped GO sheets confirming that GO is efficiently exfoliated and form high porous structures. Like TEM, FESEM also confirms the formation of the sheets. Figure 5(d) shows the EDX spectrum of GO confirming the presence of carbon and oxygen ions in GO. The element analysis 
shows $76 \%$ of carbon and $24 \%$ of oxygen, confirming that the GO powder has high purity and is oxidized because of the existence of the GO functional groups. Theoretically, the expected stoichiometric mass-percentage of carbon and oxygen are $70 \%$ and $30 \%$, respectively.

\section{Photocatalytic Dye Degradation and GO Reduction Experiments}

Figure 6 displays the photodegradation mechanism of a dye by the prepared GO sheets under the exposure of visible light. After the absorption, there is a generation of electron-hole pair, producing highly reactive species (super-oxides $\left(\mathrm{O}_{2}^{-}\right)$and hydroxyl radicals $(\cdot \mathrm{OH})$ ) that further participate in chain-reactions and break the toxic dye molecules into harmless products such as $\mathrm{CO}_{2}$, and $\mathrm{H}_{2} \mathrm{O}^{25}$.

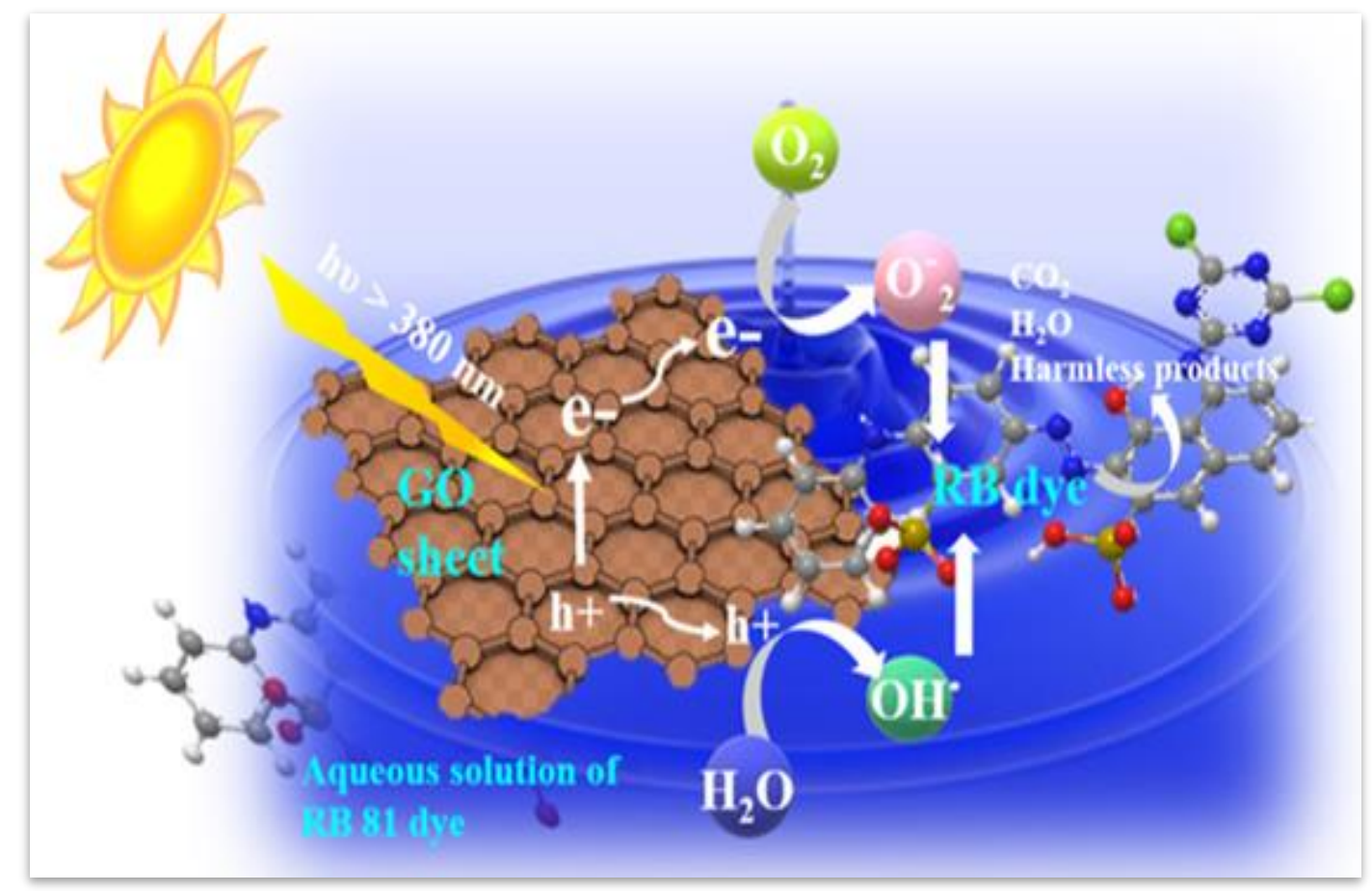

Figure 6. A schematic diagram of the dye degradation process by the synthesized GO. 
The process of dye degradation for RB81 and CR BS in the presence of GO involves five steps. (i) Visible light absorbed by GO and electrons are excited from VB to CB, and holes are generated in $\mathrm{VB}: \mathrm{GO}+\mathrm{h} v$ (incident photon) $\rightarrow \mathrm{e}^{-}(\mathrm{CB})+\mathrm{h}^{+}(\mathrm{VB})$ (ii) These photo-generated electrons and holes move to the surface of GO (iii) Participation of these electrons and holes in surface reactions like

$$
\begin{aligned}
& \text { Reduction; } \mathrm{e}^{-}(\mathrm{CB})+\mathrm{O}_{2} \rightarrow \mathrm{O}_{2}^{-} \text {. (Superoxide) } \\
& \text { Oxidation; } \mathrm{h}^{+}(\mathrm{VB})+\mathrm{H}_{2} \mathrm{O} \rightarrow \mathrm{H}^{+}+\mathrm{OH} \cdot \text { (Hydroxyl radical) } \\
& \text { Recombination of electrons and holes }
\end{aligned}
$$

(iv) Additionally, there are chain-reactions, and can be described as:

$$
\begin{aligned}
& \mathrm{O}_{2 \cdot}^{-}+\mathrm{H}^{+} \rightarrow \mathrm{HO}_{2} \cdot \\
& \mathrm{HO}_{2} \cdot+\mathrm{O}_{2 \cdot}^{-}+\mathrm{H}^{+} \rightarrow \mathrm{H}_{2} \mathrm{O}_{2} \cdot+\mathrm{O}_{2} \text { and } \mathrm{HO}_{2} \cdot+\mathrm{e}^{-}(\mathrm{CB}) \rightarrow \mathrm{HO}^{-}+H \cdot \\
& \mathrm{H}_{2} \mathrm{O}_{2}+\mathrm{h} v \rightarrow 2 \mathrm{OH} \cdot \\
& \mathrm{HO}^{-}+h^{+}(\mathrm{VB}) \rightarrow \mathrm{OH}
\end{aligned}
$$

(v) Finally, photodegradation of dyes by generated reactive species $\left(\mathrm{O}_{2}^{-}\right.$or $\mathrm{OH} \cdot$ ), namely

$$
\begin{gathered}
\mathrm{O}_{2}^{-} \text {or } \mathrm{OH} \cdot+\operatorname{dyer}(\mathrm{RB} 81 \text { and } \mathrm{CR} B S) \rightarrow \text { Dye mineralization } \\
\rightarrow \text { End Products }+\left(\mathrm{H}_{2} \mathrm{O}+\mathrm{CO}_{2}\right) \text { harmless prodcuts }
\end{gathered}
$$

Figure $7 \mathrm{a} \& \mathrm{~b}$ depict the adsorption and photodegradation efficiency of GO sheets under dark condition and visible light exposure for RB81 and CR BS respectively. Initially with a fixed dosage of GO and the concentration of each dye, the maximum adsorption equilibrium in the dark was achieved after 30 hours $(\mathrm{pH}=9)$. Figure $7(\mathrm{a})$ shows that after the dark adsorption equilibrium, the initial maximum adsorption intensity at the maximum wavelength of $581 \mathrm{~nm}$ falls from 1.21 to 0.81 for the RB81 dye. After that, on irradiating the solution (dye and GO) under visible light, the photocatalyst GO can 
degrade the dye solution completely within 90 minutes, as observed by the additional reduction of peak intensity from 0.81 to almost zero.
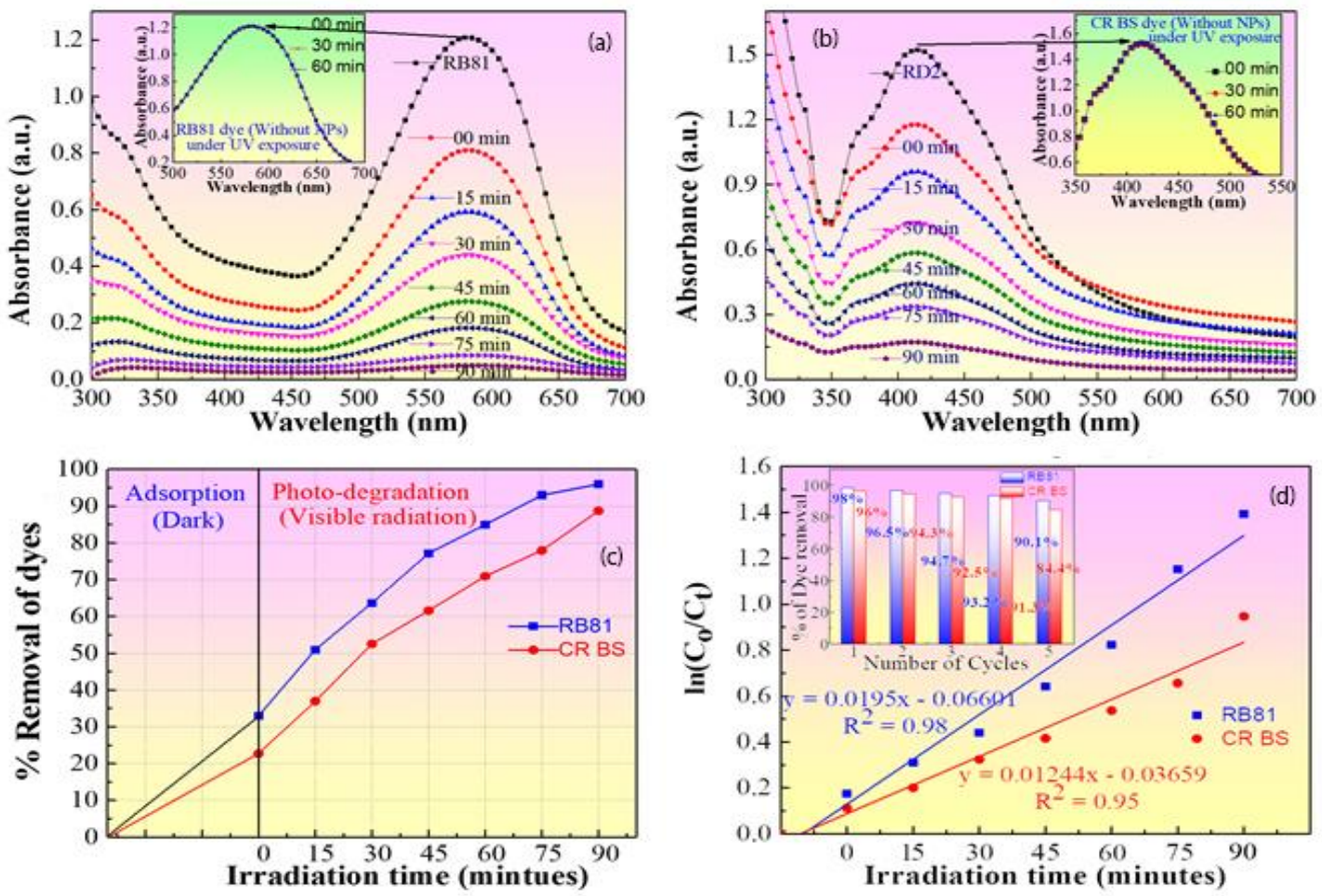

Figure 7. Photocatalytic degradation plots of (a) RB81 dye and (b) CR BS dye (c) \% removal efficiency and (d) Pseudo first order kinetics plot (inset).

The dye blue-color fades to transparent, indicating the photodegradation efficiency of GO towards RB81 dye. Similarly, Fig. 7(b) shows the UV-vis spectrum of adsorption and photocatalytic action of GO for the second dye (CR BS). The spectrum depicts the decrease of the intensity of the peak from absorbance value 1.523 to 1.177 (at $\mathrm{pH}$ of 9). The dye solution under the irradiation of visible light showed a continuous decrease in absorbance value at consistent time intervals. After 90 minutes of exposure, the dye molecules are completely degraded and the solution is decolorized.

Figure 7(c) shows the maximum removal dye percentage of GO for RB81 and CR BS. It is observed that adsorption and degradation removed $33 \%$ and $62.96 \%$ of the RB81, 
resulting in the total removal of $95.94 \%$. Similarly, it was confirmed that the removal of CR BS was $88.71 \%$ in which dark adsorption removed $22.72 \%$ of the CR BS dye and photodegradation removed the remaining $65.99 \%$.

According to Huo et al., graphene limits its application because it is $\mathrm{sp}^{2}$ hybridized, hydrophobic, and has no bandgap. Compared to graphene, GO holds different types of functional groups such as epoxy, carbonyl-hydroxyl, and carboxyl groups covalently restricted on basal planes or edges, which significantly enhances GO efficiency in the photocatalytic action ${ }^{26}$. Azim et al. observed that the sole use of GO in photocatalysis has not been outlined well. According to their photocatalytic analysis, pristine GO exhibits the decolorization of cationic MB dye up to $68.68 \%$ under sunlight ${ }^{27}$. Yeh et al. also confirmed the photocatalytic action of pristine GO by photo-catalyzing the splitting of $\mathrm{H}_{2} \mathrm{O}$ for the production of $\mathrm{H}_{2}{ }^{28}$. In this study, it was observed that the photodegradation efficiency of pristine GO toward dyes is significantly higher compared to that of previous reports.

Figure $7(\mathrm{~d})$ displays the plot $\ln \left(\mathrm{C}_{0} / \mathrm{C}_{\mathrm{t}}\right)$ against the irradiation time, which depicts the kinetic study of adsorption/degradation for RB81 and CR BS dyes by GO sheets. The kinetic curves for the degradation of RB81 and CR BS follow pseudo-first-order kinetics. The calculated values of the apparent rate constant $\left(\operatorname{Kappa}\left(\mathrm{min}^{-1}\right)\right)$ and regression coefficient $\left(\mathrm{R}^{2}\right)$ are $0.019 \mathrm{~min}^{-1}$ and 0.957 for $\mathrm{RB} 81$, and $0.012 \mathrm{~min}^{-1}, 0.98$ for CR BS dye. The evaluation of the reusability of GO for five successive series (inset; Fig. 7(d)) shows that there is a small reduction in the percentage removal of both dyes. Thus, a trivial reduction in photocatalytic activity enables GO as a potential candidate in the area of photocatalysis where reusability is critical. From the above discussion, it is well understood that the combined effect of adsorption and photodegradation by GO 
significantly influences the elimination of the two toxic dyes (RB81 and CR BS). The observed high degradation with multi dye degradation efficiency of GO might be because of its high oxygen functionalities and large surface area.

\section{Conclusion}

In this work, GO sheets were successfully synthesized by the modified Hummer's method. This work also reported the easy control over GO structural, morphological, and optical properties. The confirmation of the synthesis of GO was done by its characteristic absorption bands at $232 \mathrm{~nm}$ that originated from the $\pi-\pi^{*}$ transition of aromatic C-C bonds in the UV-vis absorption spectra. FTIR analysis showed that GO is associated with different functional groups and with different bond energies. The structural confirmation of GO is investigated by the appearance of a broad and sharp major peak at $2 \theta\left(10.35^{\circ}\right)$ corresponding to the (001) plane, indicating that the graphite is oxidized. The TEM image confirms that the sheets are thin translucent and stacked together. The SEM analysis confirms the erratically positioned wrinkled twodimensional GO sheets with irregular ends and crosswise dimensions up to a few $\mu \mathrm{m}$. It also affirms the exfoliation of graphene. Thus because of high lateral dimensions and many functional groups on the surface, the as-synthesized GO sheets are used for the degradation of CR BS and RB81 dyes. The results confirmed $95.94 \%$ and $88.71 \%$ removal of RB81 $\left(\mathrm{K}_{\text {appa }}=0.019 \mathrm{~min}^{-1}\right)$ and CR BS $\left(\mathrm{K}_{\text {appa }}=0.012 \mathrm{~min}^{-1}\right)$ dyes under visible solar light, enabling the potential use of GO for photocatalytic applications.

\section{Declaration of interest}

The authors declare there is no conflict of interest. 


\section{Acknowledgments}

The authors are thankful to the Sri Guru Granth Sahib World University, Fatehgarh Sahib for providing all facilities to conduct this research, and the SAIF laboratory of Punjab University, Chandigarh, and Sprint Testing Solutions, Mumbai, for providing the instrumentation for the characterization of samples. Author K.M. Batoo is extend their appreciation to the Deputyship for Research \& Innovation, "Ministry of Education" in Saudi Arabia for funding this research work through the project number IFKSURG-1437-030. 


\section{References}

1 Pal, S., Jana, U., Manna, P., Mohanta, G. \& Manavalan, R. Nanoparticle: An overview of preparation and characterization. Journal of Applied Pharmaceutical Science 1, 228-234 (2011).

2 Saini, R., Saini, S. \& Sharma, S. Nanotechnology: the future medicine. J Cutan Aesthet Surg 3, 32-33, doi:10.4103/0974-2077.63301 (2010).

3 Geim, A. K. \& Novoselov, K. S. The rise of graphene. Nature Materials 6, 183191, doi:10.1038/nmat1849 (2007).

4 Singh, V. et al. Graphene based materials: Past, present and future. Progress in Materials Science 56, 1178-1271, doi:10.1016/j.pmatsci.2011.03.003 (2011).

5 Novoselov, K. S. et al. Two-dimensional atomic crystals. Proc Natl Acad Sci U S A 102, 10451-10453, doi:10.1073/pnas.0502848102 (2005).

6 Novoselov, K. S. et al. Unconventional quantum Hall effect and Berry's phase of $2 \pi$ in bilayer graphene. Nature Physics 2, 177-180, doi:10.1038/nphys245 (2006).

7 Paulchamy, B., Arthi, G. \& Durai, L. A Simple Approach to Stepwise Synthesis of Graphene Oxide Nanomaterial. Journal of Nanomedicine \& Nanotechnology 06, doi:10.4172/2157-7439.1000253 (2015).

8 Das, S., Sudhagar, P., Kang, Y. S. \& Choi, W. Graphene synthesis and application for solar cells. Journal of Materials Research 29, 299-319, doi:10.1557/jmr.2013.297 (2014).

9 Sakorikar, T., Kavitha, M. K., Vayalamkuzhi, P. \& Jaiswal, M. Thicknessdependent Crack Propagation in Uniaxially Strained Conducting Graphene 
Oxide Films on Flexible Substrates. Scientific Reports 7, 2598, doi:10.1038/s41598-017-02703-2 (2017).

10 Yan, J. et al. Electrochemical properties of graphene nanosheet/carbon black composites as electrodes for supercapacitors. Carbon 48, 1731-1737, doi:10.1016/j.carbon.2010.01.014 (2010).

11 Zhang, Y., Yuan, S., Zhao, Y., Wang, H. \& He, C. Synthesis of novel yttriumdoped graphene oxide nanocomposite for dye removal. Journal of Materials Chemistry A 2, 7897-7903, doi:10.1039/C4TA01057D (2014).

12 Gupta, V., Ali, P. I., Saleh, T., Nayak, A. \& Agarwal, S. Chemical Treatment Technologies for Waste-water Recycling-an Overview. RSC Adv. 2, 6380-6388, doi:10.1039/C2RA20340E (2012).

13 Howell, S. W. et al. Graphene-Insulator-Semiconductor Junction for Hybrid Photodetection Modalities. Scientific Reports 7, 14651, doi:10.1038/s41598017-14934-4 (2017).

14 Yu, H., Zhang, B., Bulin, C., Li, R. \& Xing, R. High-efficient Synthesis of Graphene Oxide Based on Improved Hummers Method. Scientific Reports 6, 36143, doi:10.1038/srep36143 (2016).

15 Shahriary, L. \& Athawale, A. Graphene oxide synthesized by using modified Hummers approach. Renew. Energy Environ. Eng. 2 (2014).

$16 \mathrm{Li}$, Z. et al. Uranium(VI) adsorption on graphene oxide nanosheets from aqueous solutions. Chemical Engineering Journal 210, 539-546, doi:https://doi.org/10.1016/j.cej.2012.09.030 (2012). 
17 Pan, N. et al. Removal of $\mathrm{Th}^{4+}$ ions from aqueous solutions by graphene oxide. Journal of Radioanalytical and Nuclear Chemistry 298, 1999-2008, doi:10.1007/s10967-013-2660-2 (2013).

18 Gupta, B. et al. Role of oxygen functional groups in reduced graphene oxide for lubrication. Scientific Reports 7, 45030, doi:10.1038/srep45030 (2017).

19 Paredes, J. I., Villar-Rodil, S., Martínez-Alonso, A. \& Tascón, J. M. D. Graphene Oxide Dispersions in Organic Solvents. Langmuir 24, 10560-10564, doi:10.1021/la801744a (2008).

20 Drewniak, S. et al. Studies of Reduced Graphene Oxide and Graphite Oxide in the Aspect of Their Possible Application in Gas Sensors. Sensors 16, doi:10.3390/s16010103 (2016).

21 Luo, L. et al. Preparation of Graphite Oxide Containing Different OxygenContaining Functional Groups and the Study of Ammonia Gas Sensitivity. Sensors (Basel) 18, 3745, doi:10.3390/s18113745 (2018).

22 Stobinski, L. et al. Graphene oxide and reduced graphene oxide studied by the XRD, TEM and electron spectroscopy methods. Journal of Electron Spectroscopy and Related Phenomena 195, doi:10.1016/j.elspec.2014.07.003 (2014).

23 Shalaby, A. et al. Structural analysis of reduced graphene oxide by transmission electron microscopy. Bulgarian Chemical Communications 47, 291-295 (2015).

24 Stankovich, S. et al. Synthesis of graphene-based nanosheets via chemical reduction of exfoliated graphite oxide. Carbon 45, 1558-1565, doi:10.1016/j.carbon.2007.02.034 (2007). 
25 Lin, W., Yu, X., Zhu, Y. \& Zhang, Y. Graphene Oxide/BiOCl Nanocomposite Films as Efficient Visible Light Photocatalysts. Frontiers in Chemistry 6, doi:10.3389/fchem.2018.00274 (2018).

26 Hou, W.-C. \& Wang, Y.-S. Photocatalytic Generation of H2O2 by Graphene Oxide in Organic Electron Donor-Free Condition under Sunlight. ACS Sustainable Chemistry \& Engineering 5, 2994-3001, doi:10.1021/acssuschemeng.6b02635 (2017).

27 Bakhtiar Azim, M. et al. Effect of Dissolved Oxygen Content on Photocatalytic Performance of Graphene Oxide. doi: arXiv:1806.06492 (2018).

28 Yeh, T.-F., Chen, S.-J., Yeh, C.-S. \& Teng, H. Tuning the Electronic Structure of Graphite Oxide through Ammonia Treatment for Photocatalytic Generation of $\mathrm{H} 2$ and $\mathrm{O}_{2}$ from Water Splitting. The Journal of Physical Chemistry $C$ 117, 6516-6524, doi:10.1021/jp312613r (2013). 
Figures

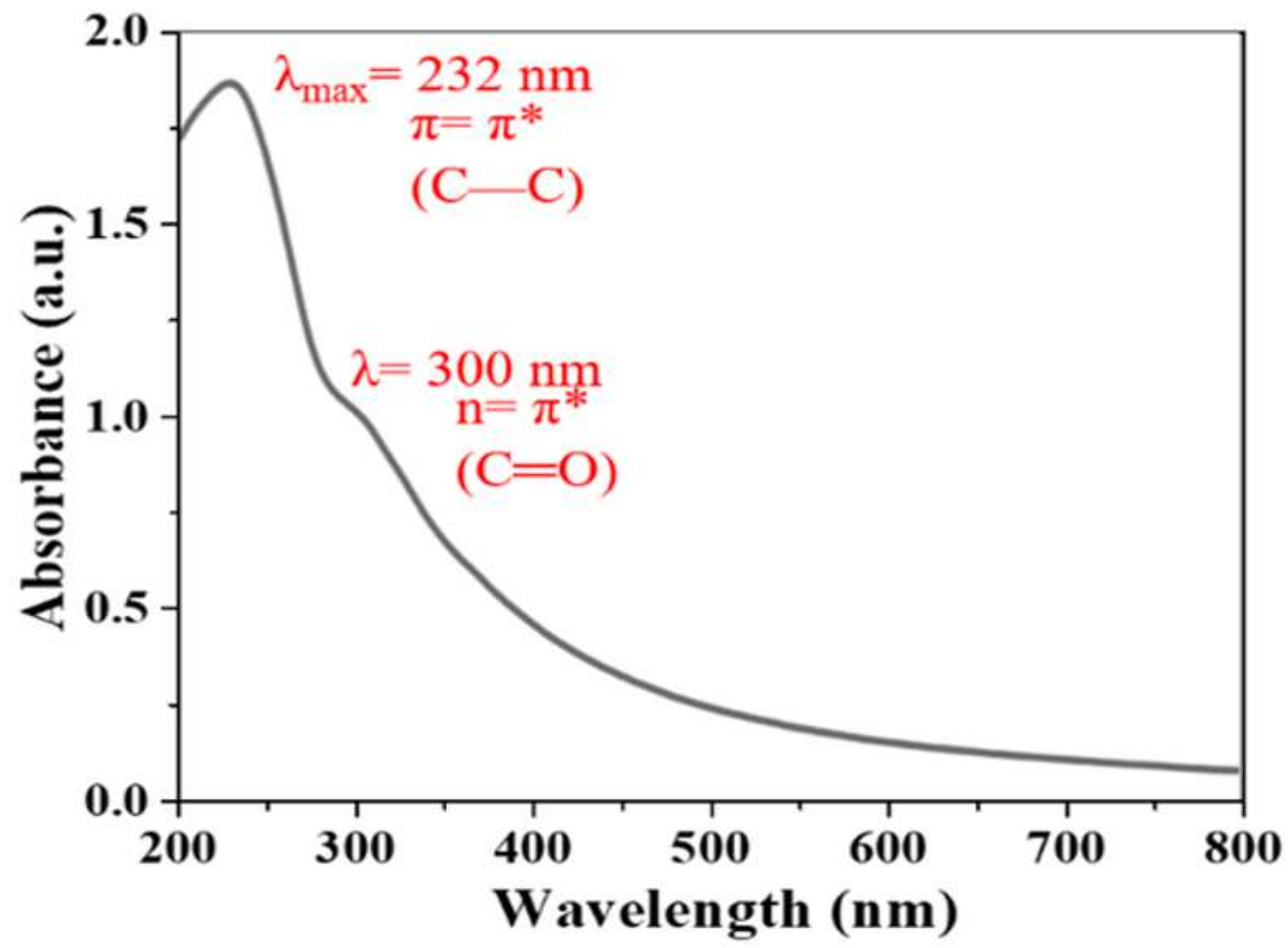

Figure 1

UV-Vis absorption spectrum of GO. 


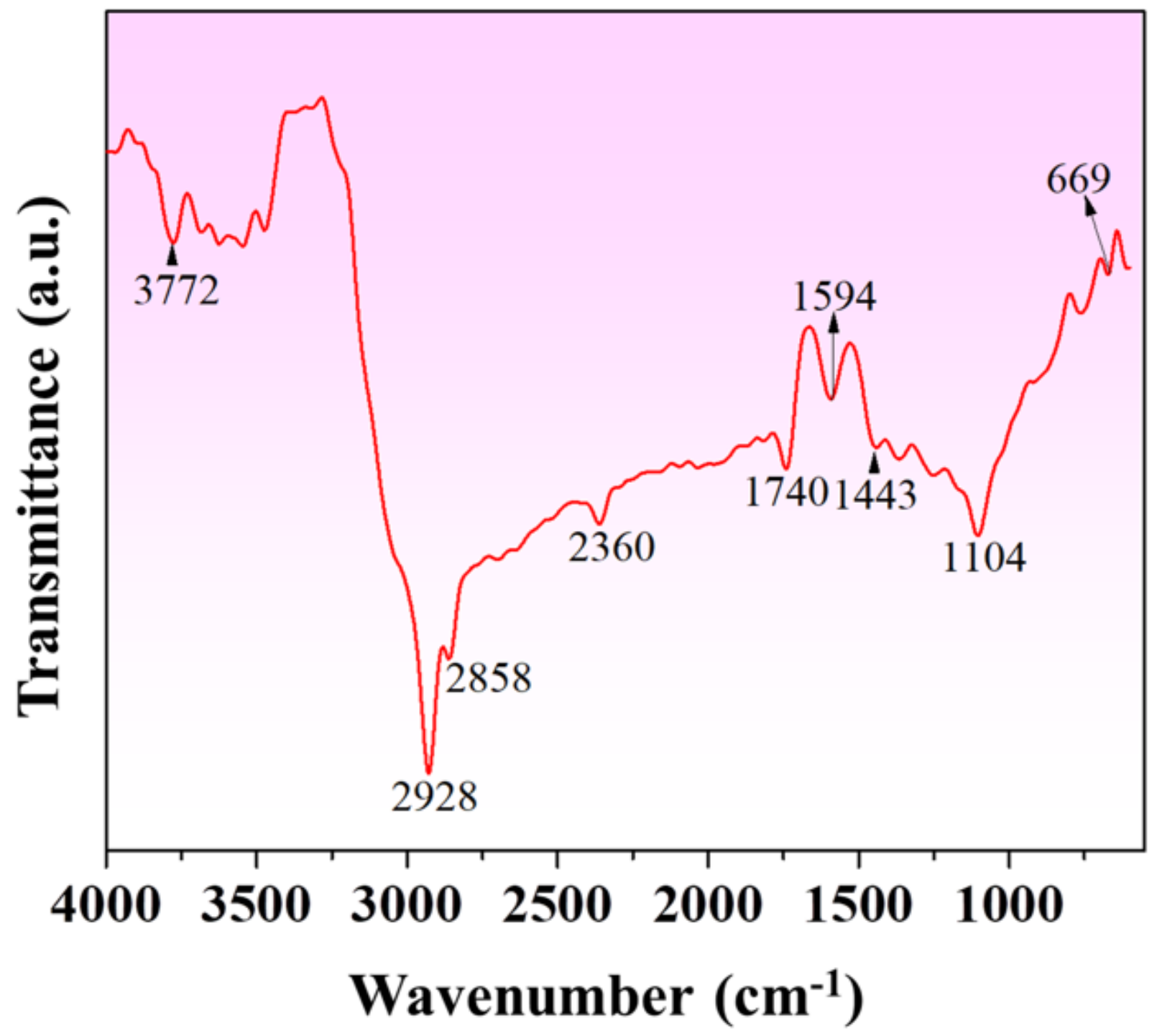

Figure 2

FT-IR spectrum of graphene oxide. 


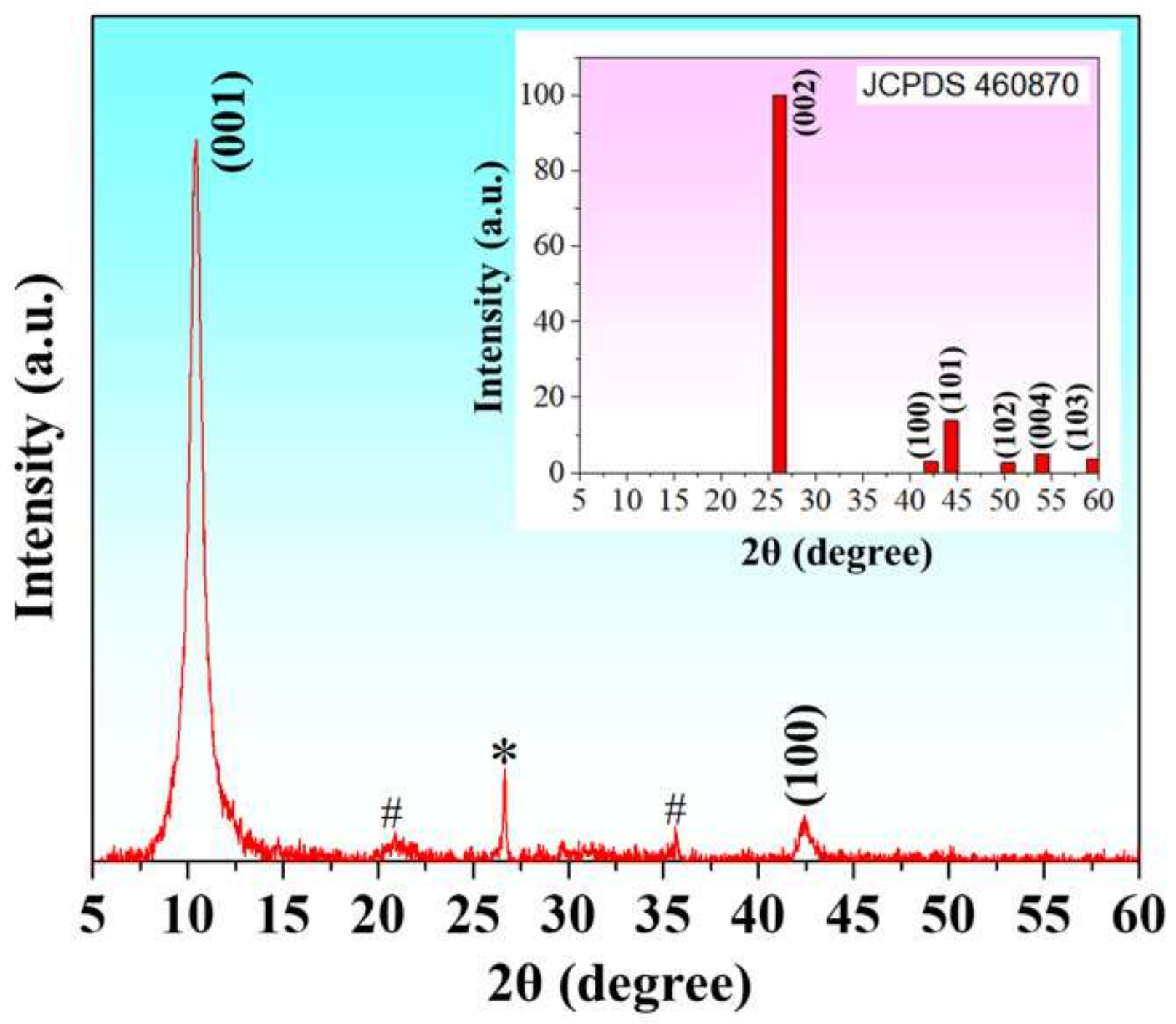

Figure 3

Powder X-Ray diffraction spectrum of the as synthesized GO. 


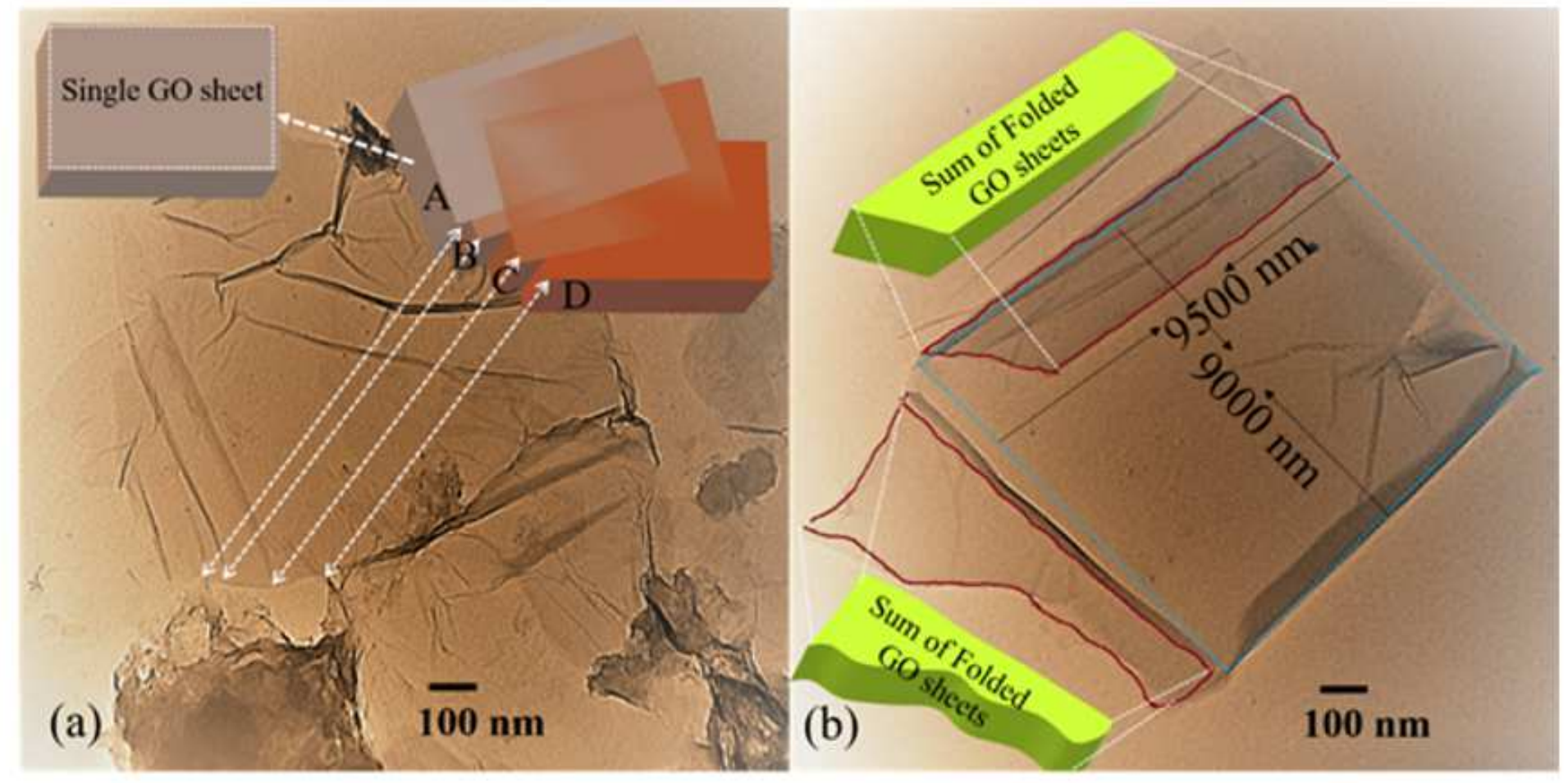

Figure 4

TEM images of graphene oxide. 


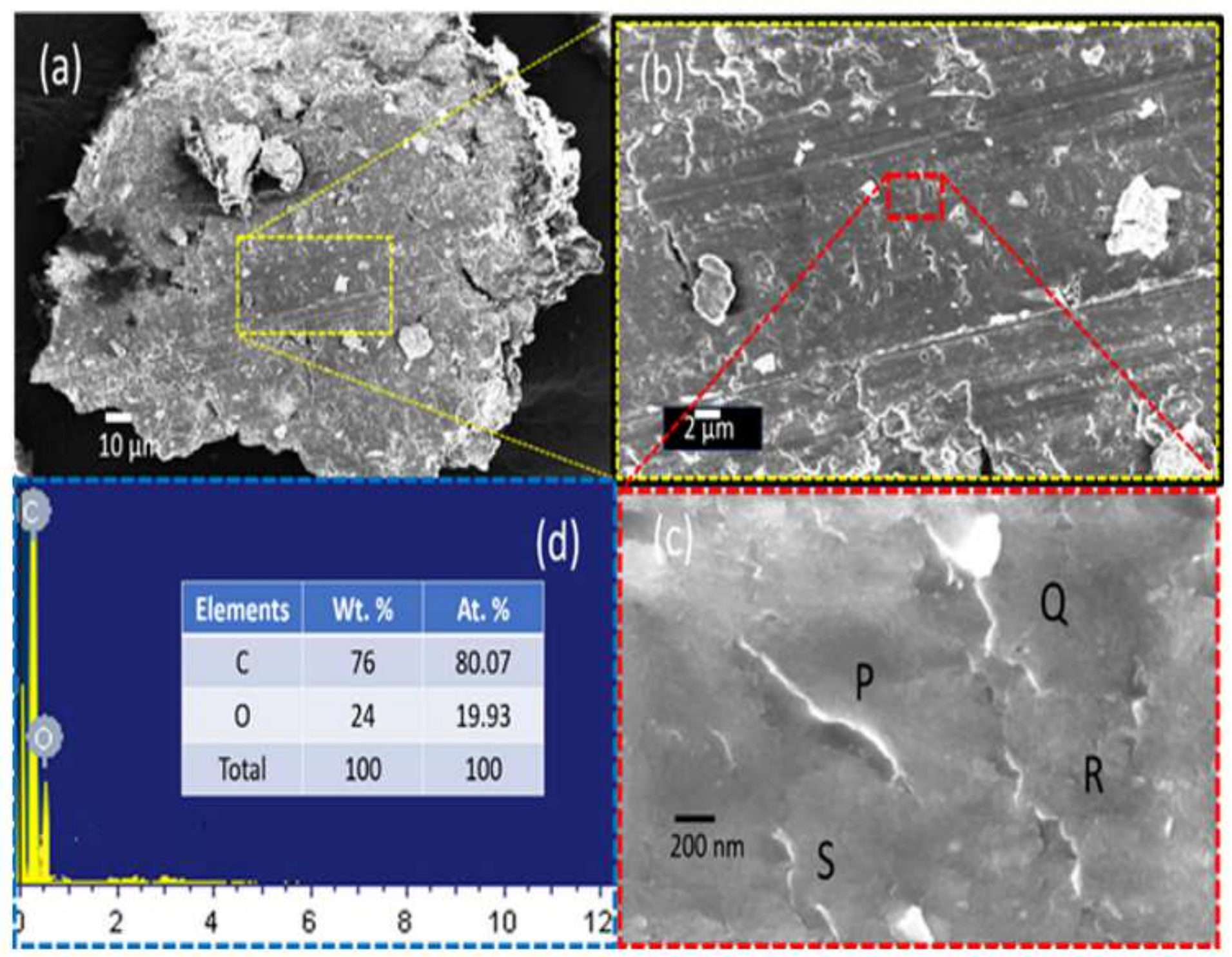

Figure 5

FESEM of Graphene Oxide by Hummers method and EDX pattern. 


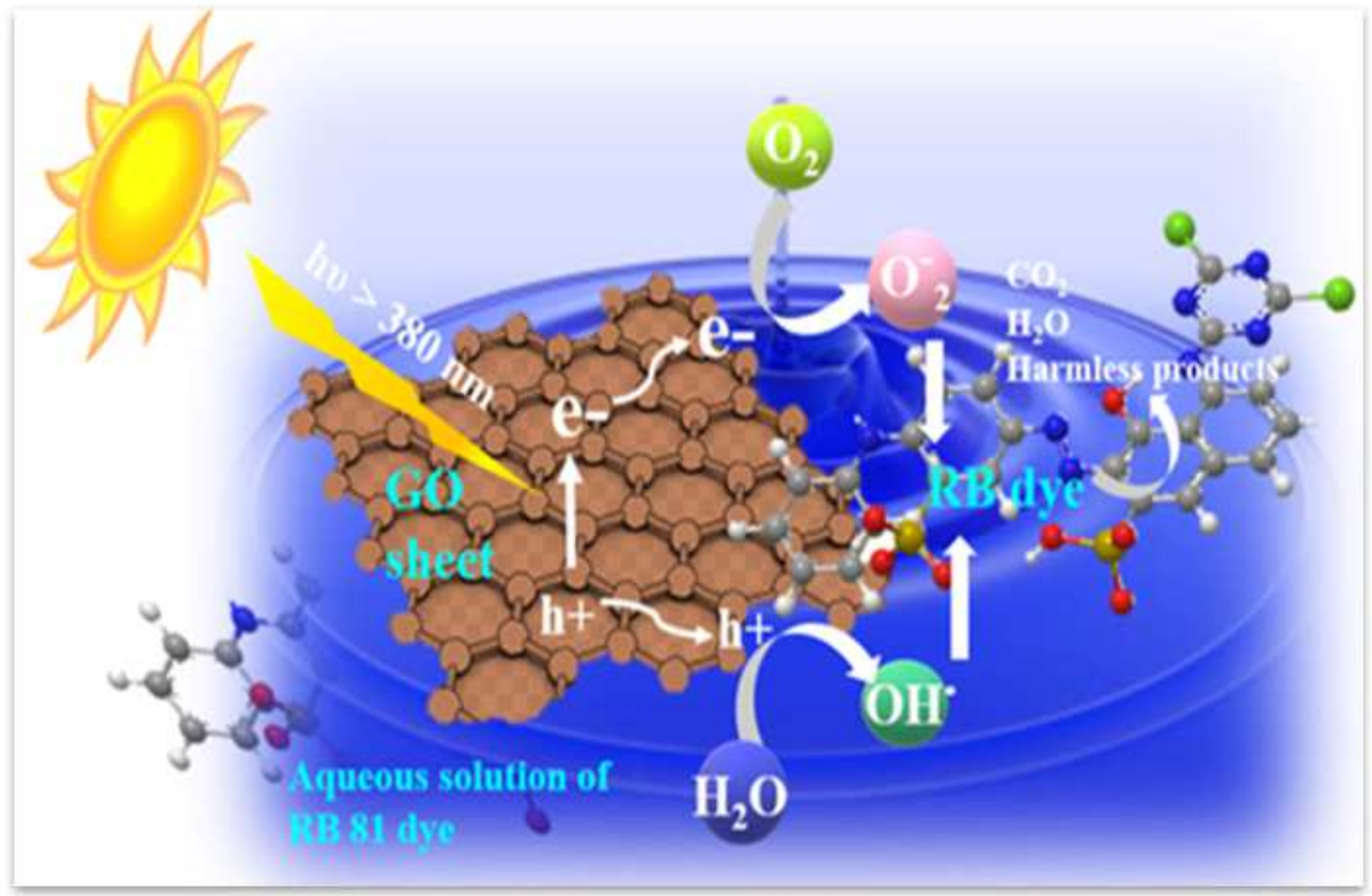

Figure 6

A schematic diagram of the dye degradation process by the synthesized GO. 

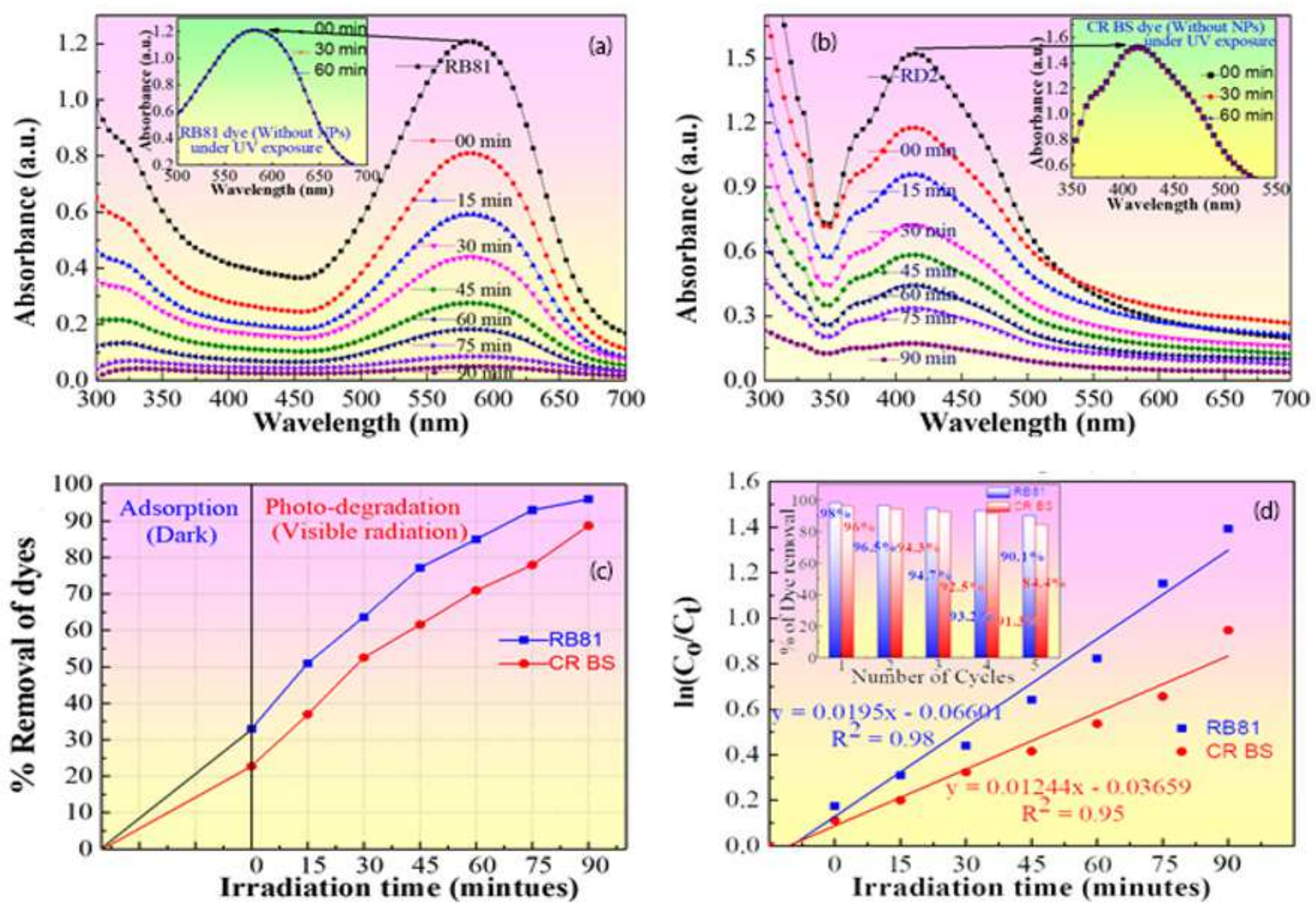

Figure 7

Photocatalytic degradation plots of (a) RB81 dye and (b) CR BS dye (c) \% 